\title{
Extracting acoustic shadowing from ultrasound image using local difference
}

\author{
Hyun Jun Park ${ }^{1}$, Kwang Baek Kim² \\ ${ }^{1}$ Division of Software Convergence, Cheongju University, Cheongju 28503, Korea \\ ${ }^{2}$ Department of Computer Engineering, Silla University, Busan 46958, Korea
}

\begin{tabular}{l} 
Article Info \\
\hline Article history: \\
Received Sep 10, 2018 \\
Revised Nov 11, 2018 \\
Accepted Nov 19, 2018 \\
\hline
\end{tabular}

Keywords:

Acoustic shadowing

Gallbladder

Gallstone

Shadow extraction

Ultrasonogram

\begin{abstract}
We propose a method for extracting acoustic shadowing from ultrasound image. If we locate the acoustic shadowing exactly then we can also extract the hyperechoic substances with a high probability. The proposed method reconstitutes an original ultrasound image to simplify calculations and uses the locally horizontal difference to extract shadow candidates. The shadow candidates are classified into start and finish point, and shadow regions are extracted by using them. The experiment results show the proposed method extracts 23 of 27 acoustic shadows from ultrasonogram efficiently and it can be used in wide applications.
\end{abstract}

Copyright $@ 2019$ Institute of Advanced Engineering and Science. All rights reserved.

\section{Corresponding Author:}

Kwang Baek Kim,

Department of Computer Engineering,

Silla University,

Busan 46958, Korea.

Email: gbkim@silla.ac.kr

\section{INTRODUCTION}

Ultrasonography is often the first imaging test done in people who have symptoms from abdomen region. It is an easy test to have done, and it uses no radiation [1]. Also ultrasonography is useful for veterinary medicine. It uses no anesthetic, and it is not expensive to diagnosis animals [2]. For a gallbladder ultrasound exam, you simply lie on a table while the doctor moves the transducer along the skin over the right upper abdomen.

Observed shape and size of tissue from ultrasonogram are used for diagnosing. Also, acoustic shadowing is also used. The acoustic shadowing used to cause by abnormal substances. That means the shadows in ultrasonogram represent diseases occasionally.

Calcification, stone, bone, and air are typical hyperechoic substances that occur post acoustic shadowing in ultrasonogram. Stated reversely, if we locate the acoustic shadowing then we can also extract the hyperechoic substances. Some hyperechoic substances such as calcification and stone are often fatal, so it must be found during ultrasonography $[3,4]$.

The acoustic shadowing has been using as an important factor in practical diagnosis. There are the field of medicine using acoustic shadowing extraction such as gallstone extraction, kidney stone predictor, calcified plaque detection, and so on [5-7]. Therefore, if we can locate the acoustic shadowing automatically, it will help the practical diagnosis in various fields of medical.

Until recently, there has been a lack of research on automatically extracting the acoustic shadowing by using medical image processing methods. There are some researches present a shadow extracting algorithm, but their algorithms are limited for specific application. The researches on extracting the acoustic shadowing from ultrasonogram can be used in wide applications are still needed. Therefore, in this paper, 
we propose a method for extracting acoustic shadowing from ultrasound image by using local difference to use in wide applications.

This paper is organized as follows. Section 2 explains a preprocessing method and shadow region extraction methods. Section 3 evaluates the performance of the proposed method using twenty images which were used in actual in-hospital diagnoses. Finally, Section 4 presents the conclusions.

\section{EXTRACTING ACOUSTIC SHADOWING}

Figure 1 shows overall process for extracting shadow regions.

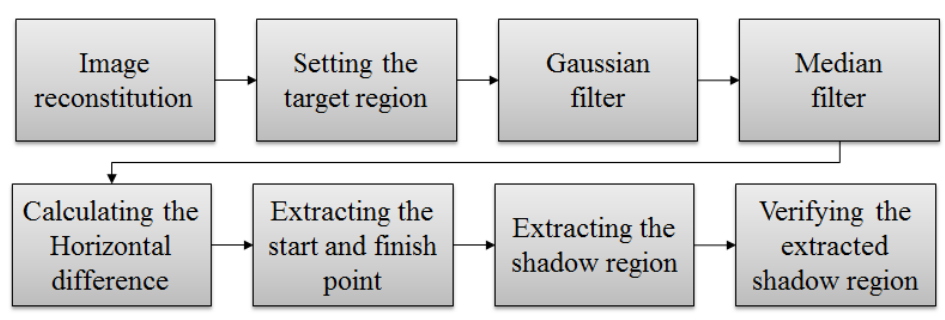

Figure 1. Overall process for shadow extraction

\subsection{Preprocessing}

Because ultrasonography uses sound waves to generate images of internal organs, the acoustic shadowing has directionality. To extract the shadow from the original image, it has to repeatedly use the sin and cos operations for calculating coordinates. It is one cause of increasing the processing time. Therefore we reconstitute the image to simplify calculations.

Figure 2 shows an example of image reconstitution. At first, as shown in Figure 2 (a), we find the location of P1 to P4 and calculate the vanishing point. After then, we reconstitute the image as shown in Figure 2 (b) using piecewise affine warping [8]. Now, we can just consider vertical direction to extract the shadowing instead of sin and cos operations.

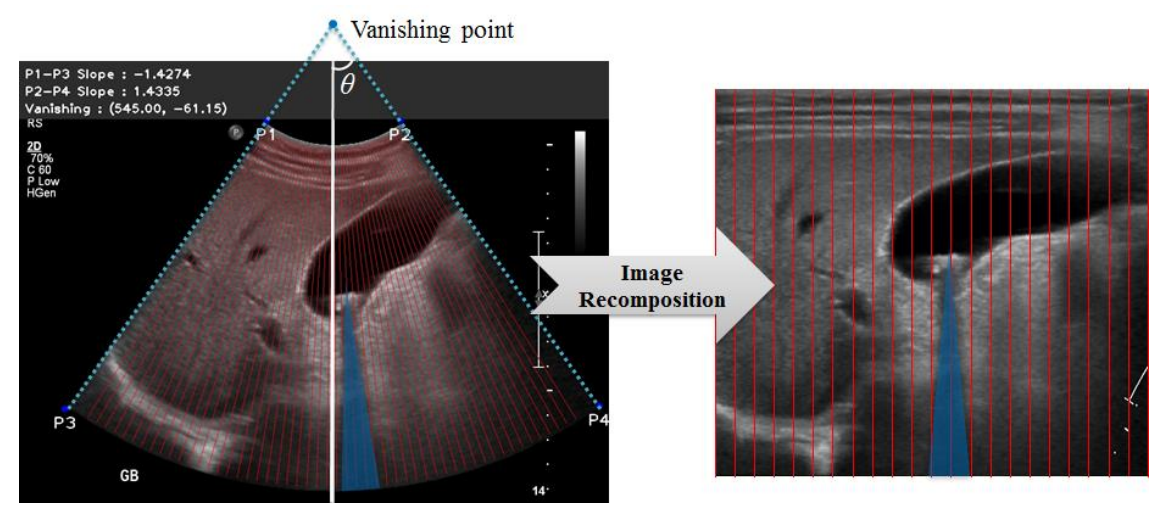

Figure 1. Image reconstitution for extracting shadow

In reconstituted image, generally, the acoustic shadowing has a vertically long and dark shape, but level of dark is not an absolute value. Therefore, we have to use the relative difference to extract the shadow.

As a preprocessing, we apply Gaussian filter to remove noise and median filter to extract shadow candidates. $3 \times 15$ size of Gaussian filter is used. It also magnifies the horizontal difference.

\subsection{Extracting the Shadow Regions}

Figure 3 shows results of the vertical and horizontal difference calculation. The vertical difference is not suitable for extracting the shadow as shown in Figure 3 (a), so we use the horizontal difference shown in 
Figure 3 (b). Even if pixels near the shadow regions are rapidly changed, adjacent pixels have similar value. Therefore we calculate the horizontal difference by using pixels ix and ix-5 ( $\mathrm{x}$ is an index of $\mathrm{x}$-axis). The regions have bigger horizontal difference than threshold is assumed to the shadow candidates.
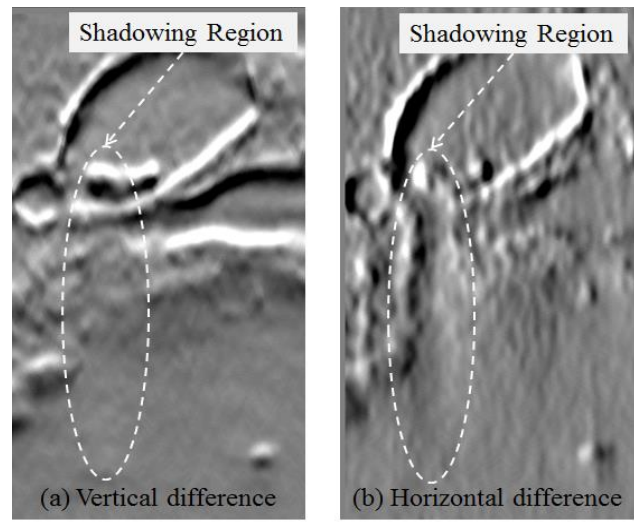

Figure 2. Vertical and horizontal difference

Figure 4 shows shadowing region extracting process. Figure 4 (a) shows region of interest for extracting the acoustic shadowing. Figure 4 (b) shows the shadow candidates. The shadow candidates are classified as shadow starting and finishing point. We define the shadow starting points as a pixel which is its vertical difference is rapidly decreased. In contrast, the finishing points are defined as a pixel that its vertical difference is rapidly increased. Figure 4 (c) shows finally results of extracting the shadow regions. The shadow can be extracted by connecting the shadow starting points and finishing points. Before extracting the shadow regions, the vertically small size of shadow candidates are removed by using size filter.
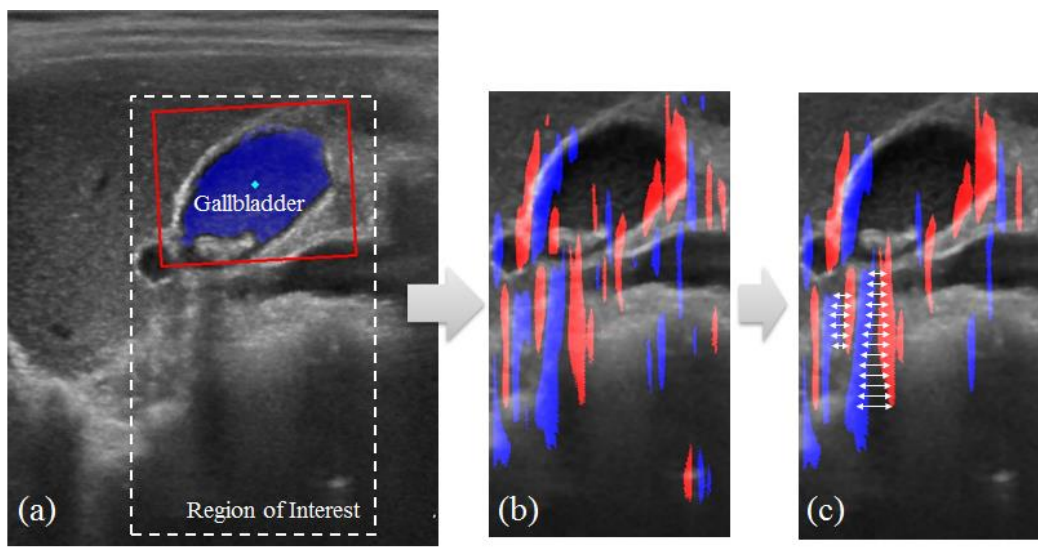

Figure 3. Process of extracting shadow regions

\section{EXPERIMENTAL RESULTS}

The software was implemented in $\mathrm{C}++$, and the Microsoft Visual 2010 Integrated Development Environment was used in the coding. Simulations were run on an IBM-compatible PC with an Intel Pentium i7-2640M CPU @ 2.80GHz with 8.0GB RAM.

Twenty ultrasound images were used for our experiment. There are total 27 shadows, and the experimental results are shown in Figure 5 and Table 1. In Figure 5 and 6, dotted lines indicate actual shadow regions and solid lines are extracted regions by the proposed method. 


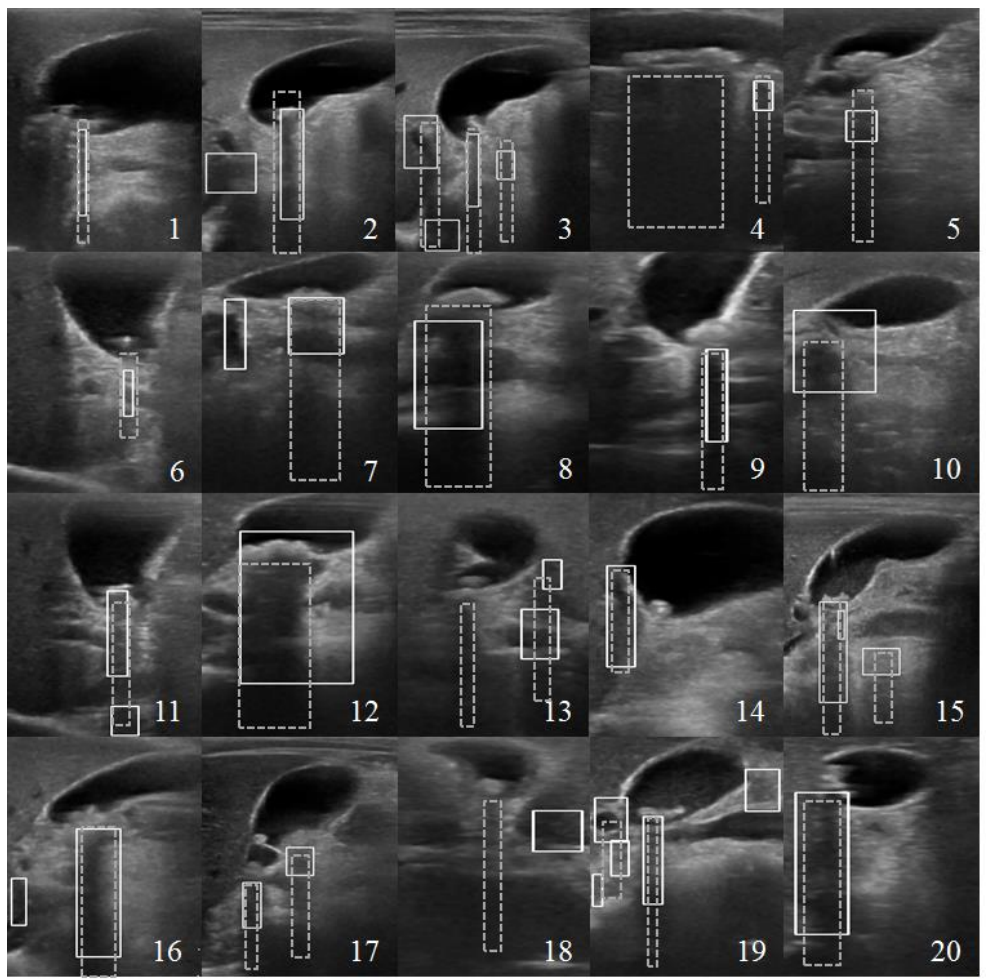

Figure 4. Results of acoustic shadow extracting

\begin{tabular}{ccc}
\multicolumn{3}{c}{ Table 1. Evaluation of the Proposed Method } \\
\hline & \multicolumn{2}{c}{ Extracting Result } \\
& Positive & Negative \\
\hline Shadow (True) & 23 (Hit) & 2 (Miss) \\
Non-shadow (False) & 5 (False alarm) & - \\
\hline
\end{tabular}

Experimental results shows 23 of 27 shadows are extracted, 5 shadows are false alarmed and 2 shadows are missed. We tried to minimize the miss cases than the false alarm to utilize the proposed method as a tool for practical diagnosis.

Figure 6 shows example of failed extractions. In 4 and 18 images, shadows have not enough horizontal difference to extract the shadows, so they are missed. In 7 and 16 images, extracted regions are similar with shadow, so they are false alarmed.

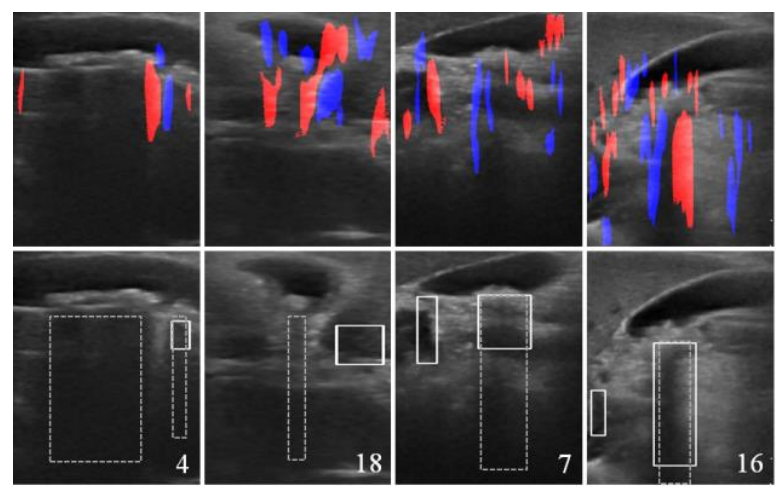

Figure 6. Example of failed extraction 


\section{CONCLUSION}

In this paper, we present a new method for extracting the acoustic shadowing from ultrasonogram. It uses the locally horizontal difference to extract the shadow candidates, and extract the shadow by connecting the shadow starting point and finishing point.

The experiment results show the proposed method extracts 23 of 27 acoustic shadows from ultrasonogram efficiently. However, there are also 5 false alarms and 2 misses. For further works, extracted shadow regions by the proposed method can be improved by verification process. It can correct the failed cases.

The proposed method can be extracting the hyperechoic substances by extracting the acoustic shadows, so it can be utilized in various ultrasonic medical image processing systems.

\section{REFERENCES}

[1] Park HJ, Kim KB, Cha EY. A new approach to locate the gallbladder from ultrasound image using intensity stretching and SOM-based color quantization. Current Medical Imaging Reviews. 2015; 11(1): 63-68.

[2] Penninck D, d'Anjou MA. Atlas of small animal ultrasonography. John Wiley \& Sons. 2013.

[3] Park HJ, Kim KB, Cha EY. Improved Method to Locate the Gallbladder in Sonogram Based on Support Vector Machine. Journal of Medical Imaging and Health Informatics. 2016; 6(7): 1566-1569.

[4] Lee FC, Dunmire B, Harper JD., Cunitz BW, Paun M, Bailey M, Sorensen MD. Ultrasound acoustic shadow width is an accurate predictor of kidney stone size. The Journal of the Acoustical Society of America. 2014; 135(4): 2267-2267.

[5] Basij M, Taki A, Yazdchi M. Automatic shadow enhancement in intra vascular ultrasound (IVUS) images. In Biomedical Engineering (MECBME), 2014 Middle East Conference on, IEEE February 2014; 309-312.

[6] Gao Z, Hau WK, Zhang H, Zhang YT. Automatic Detection of Calcified Plaque with Acoustic Shadowing. In The International Conference on Health Informatics. Springer International Publishing, January $2014 ; 197-199$.

[7] Noble JA, Boukerroui D. Ultrasound image segmentation: a survey. Medical Imaging, IEEE Transactions on. 2006; 25(8): 987-1010.

[8] Matthews I, Baker S. Active appearance models revisited. International Journal of Computer Vision, 2004; 60(2): 135-164. 